\title{
Epoxy and hydroxy fatty acids as non-volatile lipid oxidation products in oat
}

\section{Yang, Zhen}

2019-10-15

Yang , Z , Piironen , V \& Lampi , A-M 2019 , ' Epoxy and hydroxy fatty acids as non-volatile lipid oxidation products in oat ' , Food Chemistry , vol. 295 , pp. 82-93 . https://doi.org/10.1016/j.foodchem.2019.05.0

http://hdl.handle.net/10138/315058

https://doi.org/10.1016/j.foodchem.2019.05.052

cc_by_nc_nd

acceptedVersion

Downloaded from Helda, University of Helsinki institutional repository.

This is an electronic reprint of the original article.

This reprint may differ from the original in pagination and typographic detail.

Please cite the original version. 
2 Epoxy and hydroxy fatty acids as non-volatile lipid oxidation products in oat

3

4 Zhen Yang*, Vieno Piironen and Anna-Maija Lampi

5 Division of Food Chemistry, Department of Food and Nutrition, University of Helsinki, P.O. Box

6 66, FI-00014, Helsinki, Finland

7

${ }^{*}$ Corresponding author: P.O. Box 66, Agnes Sjöbergin katu 2, FIN-00014 Helsinki, Finland.

Tel.: +358456410500

E-mail: zhen.yang@ helsinki.fi (Z. Yang) 
9 The aim of the study was to investigate the occurrence and formation of peroxygenase catalysed non-volatile oxidised fatty acids (NVOFAs), especially epoxy and hydroxy fatty acids, which

11 acids from oleic and linoleic acids were present as major NVOFAs in non-heat treated (NHT) oat

14 products, and the contents increased markedly during storage. In a controlled storage experiment, 15 NVOFA contents in NHT oat flours increased to 1700-2000 $\mu \mathrm{g} / \mathrm{g}$, whereas in heat-treated samples, potentially provide off-flavours in oat. A method based on extraction of NVOFAs using accelerated solvent extraction and analysis by UHPLC-ELSD/MS was developed. Hydroxy and epoxy fatty only to $200-400 \mu \mathrm{g} / \mathrm{g}$. Epoxy fatty acids seemed to be the first products that occurred, followed by

7 hydroxy fatty acids and minor NVOFAs. The formation of NVOFAs was related to lipase catalysed

8 lipid hydrolysis and the formation of volatile lipid oxidation products. Inactivation of lipid degrading enzymes is crucial to producing stable oat products.

20

1 Keywords: Oat; peroxygenase activity; epoxy and hydroxy fatty acids; lipid oxidation; lipid hydrolysis 


\section{Introduction}

Oat (Avena sativa) is a valuable food source that provides humans and animals many nutrients, including proteins and soluble dietary fibres (Decker, Rose, \& Stewart, 2014). Oat have a higher content of lipids than most other cereal grains. Together with the activity of lipid-modifying enzymes, especially that of lipase, this may lead to off-flavour problems during processing and storing oat (Lehtinen \& Kaukovirta-Norja, 2011; Yang, Piironen, \& Lampi, 2017). When lipase comes in contact with the substrates, especially in milled oat, it begins to extensively hydrolyse acyl esters and release free fatty acids (FFAs). Generally, the FFAs are more prone to oxidation than the acyl esters, through both chemical and enzymatic pathways. However, in oat, the activity of lipoxygenase (LOX), which is responsible for the oxidation of FFAs to hydroperoxides, is low, and fatty acids are primarily subjected to autoxidation, which results in the formation of hydroperoxides (Yang et al., 2017). Oat also possess high peroxygenase (POX, EC 1.11.2.1) activity, which catalyses hydroperoxide-dependent conversion of unsaturated fatty acids into epoxy and hydroxy fatty acids (Hamberg \& Hamberg, 1996). To maintain acceptable quality in oat products, all these enzyme activities must be controlled. Oat enzymes are commonly inactivated using hydrothermal treatments, including kilning or steaming. However, some producers prefer not to use any heat treatments, e.g., to avoid changes in protein functionality.

Epoxy and hydroxy fatty acids as well as epoxy hydroxy and di- and trihydroxy fatty acids are all produced in oat using multi-step oxidation processes, with POX as the final enzyme (Hamberg \& Hamberg, 1996; Doehlert, Angelikousis, \& Vick, 2010). The formation of POX products should be avoided because some of them are thought to cause bitter off-flavours in oat products (Biermann, Wittmann, \& Grosch, 1980). In the present study, all potentially bitter POX products are called nonvolatile oxidised fatty acids (NVOFA). Oat POX catalyses intermolecular oxygen transfer from hydroperoxides to unsaturated fatty acids, which forms epoxy and hydroxy fatty acids, as well as 
intramolecular oxygen transfer from unsaturated fatty acid hydroperoxides to hydroxy-epoxy fatty acids (Hamberg \& Hamberg, 1996; Piazza, Foglia, \& Nuñez, 1999; Hanano et al., 2006). The POX pathway also includes epoxide hydrolase activity that produces trihydroxy fatty acids (Heimann \& Klaiber, 1977; Hamberg \& Hamberg, 1996; Blée 1998). Recently, two new POX activities have been found in oat (Benaragama, Meesapyodsuk, Beattie, \& Qiu, 2017).

Because they are secondary metabolites in plants, several epoxy and hydroxy fatty acids have been found in freshly milled oat seeds, including the mono-epoxides of oleic and linoleic acids, which include 9,10-epoxy-ocatadecanoic acid, 12,13-epoxy-octadec-9-enoic acid and 9,10-epoxy-octadec12-enoic acid, which together account for about $0.4 \%$ of the FFAs, according to Leonova, Shelenga, Hamberg, Konarev, Loskutov and Carlsson (2008). These authors also detected hydroxy fatty acids, including 15-hydroxy-octadeca-9,12-dienoic acid. In addition, Doehlert et al. (2010) identified 13hydroxy-octadeca-9,11-dienoic acid and 9-hydroxy-octadeca-10,12-dienoic acid in oat. Although NVOFAs have been suggested as being responsible for an undesirable bitter taste and have been associated with off-flavours (Baur, Grosch, Wieser, \& Jugel, 1977; Biermann et al., 1980), their formation in oat has been monitored in only one study (Doehlert et al., 2010), where flour from raw oat groats was stored at $37{ }^{\circ} \mathrm{C}$ for 22 weeks. At the end of storage, $5.0 \%$ of the total fatty acids were found to be epoxy acids and $1.1 \%$ were found to be hydroxy acids. The corresponding amounts in flour from steamed oat groats were less being $1.9 \%$ and $0.9 \%$, respectively, indicating that hydrothermal treatment retarded their formation to some extent. However, little is known about the formation and significance of epoxy and hydroxy fatty acids in oat products.

Finding an efficient extraction method with which to isolate NVOFAs from oat matrix without subjecting them to further reactions is challenging because lipids may be entrapped by and bound with other components, including starch and proteins. Previously, solvent extraction has been used 
to extract epoxy and hydroxy fatty acids from food matrices (Doehlert et al., 2010; Leonova et al., 2008). Accelerated solvent extraction (ASE) is a technique that provides an alternative way to extract lipids from complex food matrices using high pressure to accelerate penetration of the solvents into the samples. A gentle ASE method has been developed to extract the lipids from dry extruded pet food by limiting the extraction temperature to $40{ }^{\circ} \mathrm{C}$ (Yao and Schaich, 2014). Both gas chromatography (GC) (Doehlert et al., 2010; Jenske \& Vetter, 2007; Simsek \& Doehlert, 2014) and liquid chromatography (LC) have been used to analyse hydroxy and epoxy fatty acids (Levandi, Püssa, Vaher, Toomik, \& Kaljurand, 2009; Yang, Yang, \& Li, 2013). LC methods are becoming more commonly used to analyse epoxides (Orellana-Coca, Adlercreutz, Andersson, Mattiasson, \& Hatti-Kaul, 2005; Yang et al., 2017) and hydroxides (Yang et al., 2013; Levandi et al., 2009) because they require less-complex sample preparation.

The aim of the present study was to investigate the formation of NVOFAs in oat, focusing mainly on epoxy and hydroxy fatty acids. The study's first aim was to develop a method of analysing epoxy and hydroxy fatty acids in oat samples using ultra-high performance liquid chromatography coupled with evaporative light scattering detection or mass spectrometry (UHPLC-ELSD/MS). The second aim was to study the occurrence and formation of NVOFAs in selected heat-treated (HT) and non-heat-treated (NHT) oat samples; both included in the study to determine whether the NVOFAs were mainly formed enzymatically or chemically. The third aim was to study the formation of NVOFAs and to relate their formation to those of FFAs and volatile lipid oxidation products in a controlled storage experiment using NHT and HT oat. 


\section{Materials and Methods}

\subsection{Chemicals and standards}

Oleic and linoleic acid (purity > 99\%) were purchased from Nu-Check Prep (Elysian, MN, USA). Cumene hydroperoxide (80\%), triglyceride palmitate and 12-hydroxyoctadecanoic acid (99\%) were obtained from Sigma-Aldrich (St. Louis, MO, USA), and 9,10-epoxyoctadecanoic acid was purchased from Santa Cruz Biotechnology (Santa Cruz, CA, USA). The 12,13-epoxy-octadec-9enoic acid and the 9,10-epoxy-octadec-12-enoic acid were produced from linoleic acid as a substrate using the method developed by Yang et al. (2017). The solid phase extraction (SPE) cartridges Strata SI-1 Silica $(55 \mu \mathrm{m}, 70$ A, $500 \mathrm{mg} / 3 \mathrm{ml})$ were obtained from Phenomenex (Torrance, CA, USA). All other reagents were purchased from Sigma-Aldrich and Merck (Darmstadt, Germany). The chemicals were of pro analysis grade, and the solvents were of high performance liquid chromatography (HPLC) grade. The water used in the analyses was purified using a Milli-Q system (0.22 $\mu \mathrm{m}, \geq 18.2 \mathrm{M} \Omega \mathrm{cm}$, Millipore Corp.; Bedford, MA, USA).

\subsection{Oat samples and storage experiment}

A NHT oat flour sample provided by Raisio Group (Raisio, Finland) was stored at $35{ }^{\circ} \mathrm{C}$ for 10 weeks to allow the formation of the NVOFAs, particularly of epoxides and hydroxides, and then used as an in-house reference to verify the analytical level of the measurements. It was also used in developing the NVOFA analysis method.

The occurrence of NVOFAs was studied in five commercial oat samples that had various processing and storage histories. They included three old samples: oat flour from HT grains stored in a freezer for several years (O-HT-F1), oat flakes from HT grains stored at room temperature for more than one year (O-HT-F2) and oat powder milled from NHT grains and stored at room temperature for one year (O-NHT-P). The two other NHT oat samples included freshly milled oat 
grains that had been stored at $4{ }^{\circ} \mathrm{C}$ for several years before milling (F-NHT-F1) and freshly purchased oat flour (F-NHT-F2). The oat flakes were milled using a Knife Mill GM200 (Retsch; Haan, Germany), and the oat grains were milled using a Centrifugal Mill ZM200 (Retsch; Haan, Germany) fitted with a 0.5 -mm sieve. All the flour samples were stored in a freezer at $20{ }^{\circ} \mathrm{C}$ before analysis. To investigate the formation of NVOFAs in the two freshly milled samples, F-NHT-F1 and F-NHT-F2, a storage experiment was conducted. These samples were stored at $35^{\circ} \mathrm{C}$ for 0,6 , 12,18 and 24 weeks before being analysed for NVOFAs.

To further study the effect of HT of the oat grains on the formation of epoxy and hydroxy fatty acids, a controlled storage experiment was conducted using freshly milled flours made from the NHT and HT oat grains of two cultivars (NHT/HT-CV1 and CV2, Raisio Group; Raisio, Finland) and of NHT and HT oat grains from a mixture of cultivars (NHT-IS and HT-IS, Fazer Mills; Vantaa, Finland). All HT samples were subjected to kilning (a hydrothermal treatment) at industrial scale. The grains were milled using the method mentioned above. Subsamples of ca. $10 \mathrm{~g}$ of each of the NHT/HT-CV1 and CV2 and the NHT-IS and HT-IS materials were divided into glass bottles and stored at $40{ }^{\circ} \mathrm{C}$ for $0,1,2,3$ and 4 months. One bottle was prepared for each storage point. The NVOFAs contents were measured at each storage point.

To relate the formation of NVOFAs to lipid hydrolysis and oxidation, FFAs and volatile oxidation products were measured in selected oat samples at certain storage points. In the NHT/HT-CV1 and CV2 oat samples, lipid hydrolysis was measured at $0,1,3$ and 4 months and volatile analysis at 0,1 and 3 months. In the NHT-IS and HT-IS samples, both lipid hydrolysis and oxidation were measured at 0,1,2 and 4 months. FFAs were extracted from the selected subsamples as the NVOFAs. To analyse volatile lipid oxidation products, $1 \mathrm{~g}$ of each sample was weighed into 20-ml, 
dark brown vials that were tightly closed and stored at $40^{\circ} \mathrm{C}$. Then, at each storage point, incubations were conducted in triplicate for volatile lipid oxidation products.

\subsection{Development of method for measuring NVOFA}

\subsubsection{Extraction of lipids using ASE}

A gentle method for the extraction of NVOFAs was developed based on a study by Yao and Schaich (2014). Lipids were extracted using selected solvents and solvent mixtures in an ASE instrument (Dionex ASE-200, Dionex Corporation; Sunnyvale, CA, USA). The solvents included acetone, heptane:ethanol (2:1, vol\%:vol\%) and ethanol. About $1 \mathrm{~g}$ of each sample was weighed into 11-ml extraction cells and mixed with an equal amount of Ottawa sand (Fisher P/N S23-3), which was also used to fill up the cells. The extraction was performed using 1500 psi pressure, a cell temperature of $40{ }^{\circ} \mathrm{C}$, a cell preheating period of $5 \mathrm{~min}$ and 2 static extraction cycles of $10 \mathrm{~min}$. Then, the extracts were transferred from the collection tubes to round-bottom flasks for solvent evaporation in a rotary evaporator at $35^{\circ} \mathrm{C}$. The lipid extraction efficiencies of the three solvents were compared using GC to analyse fatty acid yields as fatty acid methyl esters $(n=6)$ (Yang et al., 2017). Because the heptane:ethanol (2:1, vol\%:vol\%) solvent mixture gave the highest fatty acid yields, it was chosen for use in further analyses. To analyse the NVOFAs, the lipid residues were dissolved in a total volume of $2 \mathrm{ml}$ of dichloromethane:heptane $(1: 9, \mathrm{vol} \%$ :vol\%) prior to the removal of triaglycerides (TAGs) using SPE purification. To analyse NVOFA, each oat sample was extracted and analysed in triplicate.

\subsubsection{Purification of NVOFAs using SPE}

The extracted lipids contained a large quantity of neutral lipids, especially of TAGs, which had to be removed before subjecting the extract to reversed-phase UHPLC analysis. The cartridges were activated using $5 \mathrm{ml}(2.5 \mathrm{ml} \times 2$ times $)$ of heptane, after which $1 \mathrm{ml}$ of the lipid extract was applied. 
Heptane:diethyl ether (9:1, vol\%:vol\%) eluent was used to remove neutral lipids from the cartridge. After testing at several volumes, a total amount of $10 \mathrm{ml}(2.5 \mathrm{ml} \times 4$ times $)$ was selected. Fractions after the first $5 \mathrm{ml}$ of the eluent (Fr 1a) and the second $5 \mathrm{ml}$ (Fr 1b) were collected separately, after which the solutions were dried with nitrogen and the residues were redissolved into $1 \mathrm{ml}$ of heptane for neutral lipid analysis (in Section 2.3.3.) to show the removal of TAGs. The NVOFAs were eluted from the cartridge using heptane:diethyl ether (1:4, vol\%:vol\%) that contained 1\% acetic acid. After testing several volumes, $5 \mathrm{ml}(2.5 \mathrm{ml} \times 2)$ was chosen as the amount to elute the NVOFAs. To analyse the NVOFAs, the collected solution was evaporated with nitrogen at $35^{\circ} \mathrm{C}$, and the residue was dissolved into $0.5 \mathrm{ml}$ of 2-propanol and $0.5 \mathrm{ml}$ of acetonitrile.

Recoveries of epoxy and hydroxy fatty acids using the ASE extraction and SPE purification steps were studied by analysing the recovery of the 12,13-epoxy-octadec-9-enoic acid, 9,10-epoxyoctadec-12-enoic acid and 12-hydroxyoctadecanoic acid that had been added to the oat flour before extraction. A solution containing about $2 \mathrm{mg}$ of the epoxides and $1 \mathrm{mg}$ of 12-hydroxyoctadecanoic acid was evaporated with nitrogen at $35^{\circ} \mathrm{C}$, after which the residue was redissolved into $1 \mathrm{ml}$ of ethanol, transferred to the cells and mixed with the 1-g oat-flour sample. In addition, oat-flour samples mixed only with $1 \mathrm{ml}$ of ethanol were used as references in each extraction batch. After the ASE and SPE processes, the recovery test samples were analysed using UHPLC-ELSD. The recovery tests were conducted in triplicate twice $(n=6)$.

\subsubsection{Analysing neutral lipid classes using NP-HPLC-ELSD}

Neutral lipid classes were determined using a normal-phase (NP)-HPLC method (Agilent 1200 HPLC system, Agilent Technologies; Santa Clara, CA, USA) and ELSD detection (Waters 2420 ELSD, Waters; Milford, MA, USA) as developed by Lampi et al. (2015) with slight modifications. The separation of lipids was achieved using a LiChrosorb diol column $(5 \mu \mathrm{m}, 3 \times 100 \mathrm{~mm}$, VDS 
Optilab Chromatographie Technik GmbH; Berlin, Germany) and gradient elution consisting of a mixture of heptane and $0.1 \%$ acetic acid (solvent $\mathrm{A}$ ) and of heptane with $0.1 \%$ acetic acid and $3 \%$ isopropanol (solvent B). The flow rate was set at $0.5 \mathrm{ml} / \mathrm{min}$ and the temperature to $25^{\circ} \mathrm{C}$. The total elution time was $50 \mathrm{~min}$, and the gradient, consisting of solvents A and B (vol\%:vol\%), was as follows: $0-3 \min (97: 3) ; 3-10 \min (97: 3$ to $0: 100) ; 10-35 \min (0: 100) ; 35-40 \min (0: 100$ to $97: 3)$; and 40-50 min (97:3). Using this method, it was possible to measure the contents of TAGs and FFAs from SPE fractions (Section 2.3.2.) and diluted ASE lipid extracts (Section 2.4.) as individual lipid classes. The injection volumes of Fr 1a and Fr $1 \mathrm{~b}$ were $10 \mu \mathrm{l}$ and those of ASE extracts were $10 \mu \mathrm{l}$ and $50 \mu \mathrm{l}$. A standard mixture of triglyceride palmitate and oleic acid was used to make second-order standard curves for quantification of TAGs and FFAs (100-5000 ng/injection). Lipid contents were given as $\mathrm{mg} / \mathrm{g}$ of the oat samples.

\subsubsection{Analysis NVOFAs using UHPLC-ELSD and UHPLC-Q-TOF}

A reversed-phase UHPLC-ELSD method for the separation and quantification of the NVOFAs was developed. Briefly, the separation of the NVOFAs was conducted using a reversed-phase Acquity $\operatorname{UPLC}^{\circledR}$ HSS T3 column $(1.8 \mu \mathrm{m}, 2.1 \times 150 \mathrm{~mm}$, Waters; Dublin, Ireland $)$ using a gradient elution that consisted of a mixture of Milli-Q $\mathrm{H}_{2} \mathrm{O}$ and $0.05 \%$ of acetic acid (solvent A) and of acetonitrile with $0.05 \%$ of acetic acid (solvent B) at a column temperature of $30{ }^{\circ} \mathrm{C}$ and a flow rate of 0.3 $\mathrm{ml} / \mathrm{min}$. The 28-min gradient with the two solvents (A and B, vol\%:vol\%) was as follows: 0-6 min (50:50 to $38.5: 61.5) ; 6-7 \min (38.5: 61.5) ; 7-14 \min (38.5: 61.5$ to $35.5: 64.5) ; 14-20 \min (35.5: 64.5$ to $10: 90) ; 20-25 \min (10: 90$ to $0: 100) ; 25-25.5 \min (0: 100) ; 25.5-26 \min (0: 100$ to $50: 50)$; and $26-$ $28 \mathrm{~min}$ (50:50). A final $4 \mu \mathrm{l}$ of the sample solution obtained from Section 2.3.2 was injected into UHPLC-ELSD and UHPLC quadrupole-time-of-flight (Q-TOF) equipment for analysis. The contents of the epoxides were calculated based on the standard curve determined using the 9,10epoxyoctadecanoic acid, while those of the hydroxy and epoxy-hydroxy compounds were 
calculated based on the standard curve determined using 12-hydroxyoctadecanoic acid. The NVOFAs were quantified using second-order standard curves (range 80-2000 ng/injection), and results were given as $\mu \mathrm{g} / \mathrm{g}$ of the oat samples.

The identification of the NVOFAs was conducted in a negative ion mode using an UHPLC-Q-TOF instrument (Acquity I class, Waters, Milford, MA, USA) that had an electrospray ionisation (ESI) interface coupled with a SYNAPT G2-Si Mass Spectrometer, as previously described (Yang et al., 2017) but with modifications. The column, eluents and elution gradients were the same as those used in the UHPLC-ELSD method described above. In addition, the instrumental settings of the MS were as follows: mass scanning range $m / z$ : 50-1200; capillary voltage: $2.5 \mathrm{kV}$; nebuliser gas flow: 6.2 bar; cone gas flow: $100 \mathrm{l} / \mathrm{h}$; source temperature: $100{ }^{\circ} \mathrm{C}$; desolvation gas flow: $1000 \mathrm{l} / \mathrm{h}$; desolvation temperature: $500{ }^{\circ} \mathrm{C}$; trap collision energy: $4 \mathrm{eV}$; ramp of MS/MS trap collision energy: 10-70 eV; trap gas flow: $2 \mathrm{ml} / \mathrm{min}$; and scan time: $0.3 \mathrm{~s}$.

\subsection{Measurement of lipid hydrolysis and volatile oxidation products}

To study the lipid hydrolysis in the oat samples, the FFAs were measured using normal phase (NP)HPLC-ELSD after extraction of the lipids using ASE (Section 2.3.1.) and a method developed earlier (Lampi et al., 2015), except that the flour samples were mixed with $1 \mathrm{ml}$ of ethanol before ASE extraction. Briefly, the lipids were extracted using acetone as the solvent at a pressure of 1000 psi and a cell temperature of $100{ }^{\circ} \mathrm{C}$. The cells were preheated for $5 \mathrm{~min}$, and the lipids were extracted using 2 static cycles of 10 min each. After extraction, the extracts were transferred to round-bottom flasks and evaporated to dryness using a rotary evaporator at $35^{\circ} \mathrm{C}$. The lipid residues were first dissolved in $10 \mathrm{ml}$ of heptane, further diluted 1:10 with heptane and then filtered through 0.45- $\mu \mathrm{m}$ filters into vials before being injected into the HPLC. The TAGs and FFAs were measured using the NP-HPLC-ELSD method described in Section 2.3.3. 
245 To study the lipid oxidation, volatile degradation products of hydroperoxides, especially hexanal 246 and 2-pentylfuran, were measured using a head-space solid-phase micro-extraction GC-MS (HSSPME-GC-MS) method developed by Damerau, Kamlang-ek, Moisio, Lampi and Piironen (2014). In brief, sample vials were incubated at $60{ }^{\circ} \mathrm{C}$ for $20 \mathrm{~min}$, and volatile compounds were extracted using a divinylbenzene-carboxen-polydimethylsiloxane (DVB/CAR/PDMS) fibre $(50 / 30-\mu \mathrm{m})$ at 60 ${ }^{\circ} \mathrm{C}$ for $30 \mathrm{~min}$. The compounds were separated using a SPB-624 column (30 m x $0.25 \mathrm{~mm}$ i.d., 1.4 $\mu \mathrm{m})$ and identified and quantified by MS using total ion chromatograms ( $\mathrm{m} / \mathrm{z}$ from $50-300)$. A porridge mixture of oat flakes and raspberry (Elovena, Raisio Group; Raisio, Finland) was milled and used as a reference sample to monitor the performance of the HS-SPME-GC-MS in each sample set. The volatile compound results were given as peak areas (counts* $\mathrm{s}^{*} 10^{6}$ ). The in-house reference gave repeatable peak area values for hexanal $\left(7.2 \pm 0.6\right.$ counts $\left.* s^{*} 10^{6}, n=6\right)$.

\subsection{Statistical analysis}

All results were given as mean values \pm standard deviations (SD) of triplicate samples. Figures were drawn using OriginPro 2018 (OriginLab Corporation; Northampton, MA, USA).

\section{Results and Discussion}

\subsection{Analysis of the NVOFAs from oat lipid extracts} used to extract the NVOFAs. The removal of the TAGs and a large portion of the FFAs from the lipid extracts was achieved using SPE fractionation (data not shown). Using the heptane:diethyl ether mixture (9:1, vol\%:vol\%), TAG residues were no longer detected in the second 5-ml elution of the silica cartridge. NVOFAs were completely eluted from the cartridge using $10 \mathrm{ml}$ of the 
heptane:diethyl ether mixture (1:4, vol\%:vol\%) containing $1 \%$ of acetic acid, which was shown by the high rates of recovery of the epoxy and hydroxy fatty acids that had been added to the oat sample prior to ASE extraction. The recovery values $(n=6)$ for the 12,13-epoxy-octadec-9-enoic acid and the 9,10-epoxy-octadec-12-enoic acid were $89 \%$ and $90 \%$, respectively, and $96 \%(\mathrm{n}=6)$ for the 12-hydroxyoctadecanoic acid. In addition, the method developed was repeatable and stable during the entire experiment, because the oat in-house reference sample analysed in every batch of samples provided repeatable total quantities of NOVAFs of $1964 \pm 185 \mu \mathrm{g} / \mathrm{g}$ of flour $(\mathrm{n}=9)$ after being subjected to UHPLC-ELSD analysis, as presented below.

All the NVOFAs extracted from the in-house reference oat sample and collected after SPE fractionation were separated within the elution time of 28 min using UHPLC-ELSD (Fig. 1), with two compounds apparently co-eluting at a retention time of $9.2 \mathrm{~min}$. To identify the products, UHPLC-Q-TOF with ESI in the negative mode and the relative retention times $\left(\mathrm{R}_{\mathrm{r}}=t_{\text {compound }} / t_{\text {epoxy }}\right.$ standard) were used, and quantitative analysis using ELSD as the detector was used. All the compounds produced deprotonated molecular precursor ions $[\mathrm{M}-\mathrm{H}]^{-}$, and together with their fragmentations, each NVOFA (C1-C7, Fig. 1) produced a characteristic spectrum (a-g, Fig. 2). The major characteristic fragments of $\mathrm{m} / \mathrm{z}>150$ were used in the structural identification of the compounds. Each compound had a fragment of $\mathrm{m} / \mathrm{z}, 18$ less than the precursor ion, indicating that a molecule of water was always lost during fragmentation, forming ion $\left[\mathrm{M}-\mathrm{H}-\mathrm{H}_{2} \mathrm{O}\right]^{-}$. In all the spectra, either the $[\mathrm{M}-\mathrm{H}]^{-}$ion or the $\left[\mathrm{M}-\mathrm{H}-\mathrm{H}_{2} \mathrm{O}\right]^{-}$ion had the highest intensity, that is, the base peak.

The earliest eluted NVOFAs (C1 and C2, Fig. 1) possessed the biggest $m / z$ deprotonated molecular ions, which were $m / z 313.2$ and 311.2 (Figs. 2a, b), respectively, indicating that they had more oxygen, such as in hydroxy, epoxy or hydroperoxy groups, in their structures than did other 
compounds. $\mathrm{C} 1$ produced a fragment $m / z 183.1$ (Fig. 2a) that had an intensity of $>90 \%$ relative to the base peak, and C2 produced fragments $m / z, 155.1,171.1,199.1$ and 211.1, which had intensities of $10-40 \%$ (Fig. 2b). Based on the deprotonated molecular ion and its fragmentation, $\mathrm{C} 1$ was identified as 12,13-dihydroxy-octadec-9-enoic acid $\left(C 1, \mathrm{R}_{\mathrm{r}}=0.301\right.$, Fig. 1$)$, as presented by Newman, Watanabe and Hammock (2002) and Levandi et al. (2009). In line with the results of their studies, the present study tentatively identified a minor peak eluting close after $\mathrm{C} 1$ as 9,10 dihydroxy-octadec-12-enoic acid, based on its deprotonated molecular ion $(\mathrm{m} / \mathrm{z}$ 313.2) and its major fragment $(\mathrm{m} / \mathrm{z}$ 201.1). Because it was present in such a low quantity, it was not included in the study. Hamberg and Hamberg (1996) suggested that 12,13-dihydroxy-octadec-9-enoic acid was hydrolysed from 12,13-epoxy-octadec-9-enoic acid as a minor product by soluble epoxide hydrolase and that the hydrolase preferred the 12,13-epoxy fatty acid isomer as the substrate.

C2 was tentatively identified as a hydroxy-epoxy fatty acid and apparently contained a mixture of 13-hydroxy-9,10-epoxy-octadec-11-enoic acid and 9-hydroxy-12,13-epoxy-octadec-10-enoic acid $\left(C 2, R_{r}=0.347\right.$, Fig. 1). The MS contained typical fragments for each of the isomers: the $\mathrm{m} / z .155 .1$ ion can form after scission of the carbon-carbon bond of the epoxide group at position 9,10 and the $m / z 193.1$ ion after dehydration of the scission product of the carbon-carbon bond of the epoxide group at position 12,13, as suggested by Huang and Schwab (2012) and Yuan, Majchrzak-Hong, Keyes, Iadarola, Mannes and Ramsden (2018). Epoxy-hydroxy fatty acids are POX-catalysed intramolecular epoxidation products of hydroperoxides that contain a double bond and that have been suggested as forming in oat seeds (Piazza et al., 1999; Hanano et al., 2006). The identification of the structures of $\mathrm{C} 1$ and $\mathrm{C} 2$ was further supported by their retention order in reversed phase (RP)-UHPLC (Yuan et al., 2018). 
The largest peak of the NVOFA chromatogram that eluted at 9.2 min consisted of two partly coeluting compounds (C3 and C4, Fig. 1). Two different mass spectra were obtained (Figs. 2c, d). The two compounds had the same molecular ion $\mathrm{m} / \mathrm{z}, 295.2$ and a fragment ion $\mathrm{m} / \mathrm{z}, 195.1$, but the intensity of the $\left[\mathrm{M}-\mathrm{H}-\mathrm{H}_{2} \mathrm{O}\right]^{-}$at $m / z 277.2$ was the highest in $\mathrm{C} 4$ (Fig. 2d) and much lower in $\mathrm{C} 3$ (Fig. 2c). Another difference was fragment ion $\mathrm{m} / \mathrm{z}$ 171.1, which occurred in the spectrum of $\mathrm{C} 4$ and was only minor in C3. The compounds were identified as hydroxy fatty acid regio-isomers: the first was 13-hydroxy-octadeca-9,11-dienoic acid (C3, $\mathrm{R}_{\mathrm{r}}=0.521$, Fig. 1) and the second was 9-hydroxyoctadeca-10,12-dienoic acid $\left(C 4, R_{r}=0.526\right.$, Fig. 1). The spectra were typical for unsaturated hydroxyl fatty acids showing the presence of the hydroxyl group (Murphy, 2015). The conjugated mono-hydroxy compounds $\mathrm{C} 3$ and $\mathrm{C} 4$ are typical POX-catalysed oxidation products of linoleic acid hydroperoxides, as presented by Hamberg and Hamberg (1996). Co-elution of the two isomers has also been observed when using RP-HPLC (Schneider, Schreier, \& Herderich, 1997).

The next 3 NVOFAs $\left(C 5, R_{r}=0.779 ; C 6, R_{r}=0.797 ; C 7, R_{r}=1.000 ;\right.$ Fig. 1) were identified as mono-epoxy fatty acids. C5 and C6 had deprotonated molecular ions $[\mathrm{M}-\mathrm{H}]^{-} \mathrm{m} / \mathrm{z}$ 295.2, and C7 had $[\mathrm{M}-\mathrm{H}]^{-} \mathrm{m} / \mathrm{z} 297.2$ (Figs. 2e, f, g), indicating that C5 and C6 were monounsaturated epoxy fatty acids and that $\mathrm{C} 7$ was a saturated one. The fragmentation presented in the spectra was comparable to that presented earlier (Orellana-Coca et al., 2005; Murphy, 2015; Yang et al., 2017). Thus, C5, C6 and C7 were identified as 12,13-epoxy-octadec-9-enoic acid, 9,10-epoxy-octadec-12-enoic acid and 9,10-epoxyoctadecanoic acid, respectively. C5 and C6 are typical POX catalysed oxidation products of linoleic acid and C7 of oleic acid, which all are typical epoxy fatty acids produced by POX catalysis (Hamberg \& Hamberg, 1996; Yang et al., 2017).

Overall, the method developed in the present study was able to quantitatively analyse several NVOFAs from the oat matrix, which had not yet been done. This method enables following their 
formation and degradation and understanding of the reaction routes that POX activity can catalyse 344 in oat during storage.

\subsection{Occurrence of the NVOFAs in flours from the NHT and HT oat grains}

The method developed was first used to study the occurrence of NVOFAs in selected commercial oat products. There were NVOFAs in the old NHT oat powder (O-NHT-P), the freshly milled flour from old NHT oat grains (F-NHT-F1) and the freshly purchased NHT oat flour (F-NHT-F2) (Table 1a). Although the amounts of NVOFAs differed among these NHT samples, all contained hydroxy fatty acids from linoleic acid (C3 and C4) and epoxy fatty acids from linoleic and oleic acids (C5C7). The same epoxy fatty acids had been found at similar levels in 33 oat accessions, although the hydroxy fatty acids measured in that study were different (Leonova et al., 2008). Doehlert et al. (2010) also found 0.25 mole\% of the epoxy fatty acids of total fatty acids, which was also comparable to the level found in the present study. In the two HT oat samples (Table 1a), no NVOFAs were detected. This clearly indicates that the NVOFAs are likely formed via the POX enzyme catalysed lipid oxidation pathway in NHT oat and that formation may already have begun in whole grains to some extent.

The formation of NVOFAs was further studied in two samples (F-NHT-F1 and F-NHT-F2) during a 24-week storage experiment at $35^{\circ} \mathrm{C}$ (Table 1a). Although the F-NHT-F1 and F-NHT-F2 had originally had comparable amounts of NVOFAs $(350 \pm 11 \mu \mathrm{g} / \mathrm{g}$ and $284 \pm 7 \mu \mathrm{g} / \mathrm{g}$, respectively) and the amounts increased during storage, the distribution of products differed greatly between the two materials. In both, during the first 6 weeks, the total amount of NVOFAs increased almost 4-fold, and the peak values were reached after 18 weeks. Thereafter, the amounts of NVOFAs dropped sharply to about $1100-1200 \mu \mathrm{g} / \mathrm{g}$ of flour by the end of the storage period. The original quality and the storage history of the two flours (F-NHT-F1 and F-NHT-F2) differed, and their profiles of 
NVOFAs changed in different ways. In the F-NHT-F1, both hydroxy fatty acids and epoxy fatty acids dominated until 18 weeks of storage, after which the amount of epoxy fatty acids decreased. In the F-NHT-F2, after 12 weeks of storage, the amounts of hydroxy and epoxy fatty acids and of hydroxy-epoxy-octadecenoic acid increased markedly, and the latter was the major product at the end of the storage period. In both flours, dihydroxy-octadecenoic acid formed to a small extent $(<$ $100 \mu \mathrm{g} / \mathrm{g})$. Its formation and that of hydroxy-epoxy-octadecenoic acid have not previously been detected in stored oat flour, but only in experiments using enzyme incubation. The decreasing amounts of NVOFAs indicated that either the POX activity had decreased, the products had reacted further and/or the availability of unsaturated fatty acids as substrates had decreased. To better understand the formation of NVOFAs and to relate it to other degradation reactions in lipids, a controlled study using both NHT and HT oat flours was conducted.

\subsection{Formation of NVOFAs in the flours from the NHT and HT oat grains}

In the present study, all materials were freshly milled and subjected to controlled storage conditions, and NVOFAs, the indicators of lipid hydrolysis and the formation of volatile oxidation products all were measured at regular intervals. NVOFAs formed in all oat samples during storage at $40{ }^{\circ} \mathrm{C}$, but the amounts differed greatly between flours made from NHT (Table 1b) and HT oat grains (Table 1c). Two flours made from NHT oat grains contained small amounts of the primary POX products, that is, epoxy and hydroxy fatty acids (57 $\pm 2 \mu \mathrm{g} / \mathrm{g}$ and $112 \pm 1 \mu \mathrm{g} / \mathrm{g}$, respectively), at the beginning of the storage experiment, indicating that NVOFAs may form soon after milling. In contrast, in one flour, no NVOFAs were measured. In all three NHT samples, the amounts of NVOFAs increased to their peak values of $1700-2000 \mu \mathrm{g} / \mathrm{g}$ at two months of storage, after which the levels began to decrease to $700-1100 \mu \mathrm{g} / \mathrm{g}$ by the end of the four months of storage. It is worth noting that in the first experiment, the two NHT oat flours (F-NHT-F1 and F-NHT-F2) reached their peak amounts of NVOFAs much later (at 18 weeks) than in the controlled experiment, which might indicate that the 
POX activities in the first experiment had already begun to decrease during their unknown storage history. In the controlled experiment, much smaller amounts of NVOFAs were formed in the flours from HT oat grains than in those from NHT oat grains. The NHT-CV1 sample, which contained no NVOFAs at the beginning, contained the highest amount after four months of storage. In the HT-IS sample, the total amount of NVOFAs remained stable at $165-178 \mu \mathrm{g} / \mathrm{g}$ after one month of storage, and in the HT-CV1 and HT-CV2 samples, the levels increased to $253 \pm 10 \mu \mathrm{g} / \mathrm{g}$ and $398 \pm 37 \mu \mathrm{g} / \mathrm{g}$, respectively, at three months of storage, after which they decreased to $98 \pm 1 \mu \mathrm{g} / \mathrm{g}$ at four months of storage. The present study clearly showed that using HT to inactivate lipid-modifying enzymes, particularly POX, is an efficient way to inhibit the formation of NVOFAs in oat. During the storage experiment, their contents in the HT oat samples were always $<20 \%$ of the amounts in the corresponding NHT oat samples.

The NHT oat samples always contained more epoxy fatty acids than hydroxy fatty acids, but after the highest NVOFA values were reached, the proportion of hydroxy fatty acids increased. The amount of epoxy-octadecanoic acid was always greater than the sum of epoxy-octadecenoic acids, indicating that oleic acid was as effective as linoleic acid in accepting oxygen from hydroperoxides through POX catalysis. Because only hydroxy-octadecadienoic acids were found and not hydroxyoctadecenoic acids, it was apparent that linoleic acid hydroperoxides were the co-oxidating species in the POX-catalysed reaction. Only modest amounts of hydrolysis products of epoxides, including dihydroxy-octadecenoic acids, and the intramolecular oxygen transfer reaction products of linoleic acid hydroperoxides, including hydroxy-epoxy-octadecenoic acids, were formed. These minor products were detected in NHT-CV1 samples only at the end of storage, whereas in the other samples, they were measured after one or two months of storage. In the flours made from HT grains, epoxy fatty acids were the only products detected until three months of storage, after which small amounts of hydroxy-octadecadienoic acids were present in the HT-CV1 and HT-CV2 
samples. This, along with the small total amounts of NVOFAs, indicates that peroxygenation was present in much smaller quantities in the HT materials than in the NHT ones.

In the present study, we showed a clear trend in the formation and degradation of NVOFAs in oat flours during storage. To date, only one study has reported increase of epoxy and hydroxyl fatty acids in oat grains (Doehlert et al., 2010). In that study, the epoxy fatty acid content increased 20fold in raw oat during storage for 22 weeks at $37{ }^{\circ} \mathrm{C}$, and the hydroxy acids content was only $20 \%$ of that of the final epoxy fatty acid content. However, the results of that study did not show what happened during storage because it analysed NVOFAs only at the end of storage, and, as the present study showed, the profile of NVOFAs changes markedly over time. Doehlert et al. (2010) also showed that steaming before storing retarded increases in levels of epoxy and hydroxy fatty acids by $60 \%$ and $17 \%$, respectively, which was much less than the effect of HT in the present study. Apparently, the HT the present study was more effective than that in their study at inhibiting POX.

To better evaluate the bitterness caused by NVOFAs, it would be interesting to know their threshold values, but unfortunately, these not available. However, it has been found that hexane extract of HT oat exhibits less bitterness (Günther-Jordanland, Dawid, Dietz, \& Hofmann, 2016), which could have derived from the NVOFA levels. Unfortunately, that study did not include NHT oat, which could have supported the importance of NVOFAs as a cause of bitterness.

\subsection{Relating the formation and degradation of NVOFAs in flours made from NHT and HT oat} grains to lipid hydrolysis and volatile lipid oxidation products

Since oat POX prefers free fatty acids over esterified fatty acids as substrates (Yang et al., 2017), the occurrence of free fatty acids was studied to relate their formation to that of NVOFAs. In addition, free fatty acids enhance lipid oxidation, including both LOX-catalysed oxidation and autoxidation, which is a prerequisite for NVOFA formation. The neutral lipids of freshly milled 

higher in the HT samples, which did not contain any FFAs, whereas the NHT samples already had 1-7 mg/g of FFAs at the beginning of storage, indicating that lipid hydrolysis had begun during milling. HT of oat grains efficiently inhibited lipid hydrolysis, as the content of FFAs increased only to $2 \mathrm{mg} / \mathrm{g}$ during storage, while in the NHT samples, the FFA levels had already begun to increase during the first month of storage, and the highest level reached was $42 \mathrm{mg} / \mathrm{g}$ in NHT-CV2 after three months of storage. Of the three NHT materials, NHT-CV1 was the most resistant to lipid hydrolysis, as indicated by the fact that it had the lowest FFA content and the highest TAG content, results that are in line with the slower formation of NVOFAs. Only after four months had the TAGs degraded to very low levels in NHT-CV1, while this occurred one and two months earlier in NHT-

Lipid oxidation, measured as the contents of hexanal and 2-pentylfuran, was intensive in the NHT materials and only minimal in the HT materials during the four months of storage (Fig. 4). The formation of volatile oxidation products showed that the NHT-IS sample oxidised more than the others, and their amounts in this sample reached the highest values after two months of storage, after which they levelled off and began to decrease. This occurred at the same time that the sample's TAGs were completely hydrolysed, and that the NVOFAs reached their highest amounts. After this, the amount of the NVOFAs, particularly of epoxy-fatty acids, began to decrease. indicator of enzymatic lipid oxidation and a product of linoleic acid 9-hydroperoxide (Ho \& Chen, 1994; Lampi et al., 2015), was a little greater in the NHT-CV2 sample than in the NHT-CV1 one, possibly indicating that the NHT-CV2 material had more enzymatic activity than the NHT-CV1 
material. In accordance, there were already more NVOFAs in the NHT-CV2 sample at the beginning of the storage experiment. In addition, the NHT-CV2 sample formed more dihydroxy octadecenoic acid and hydroxy-epoxy-octadecenoic acids, and its total amount of NVOFAs remained higher than the total in the NHT-CV1 sample as long as substrates were present. All the NHT samples always had higher 2-pentylfuran content than hexanal content, indicating the importance of enzymatic oxidation. The inhibition of enzymes during heat treatment played a major role in the lipid oxidation of the flours made from the HT oat grains, as measured as the levels of hexanal and 2-pentylfuran. The volatiles in the freshly milled samples likely contributed to the natural flavour of the oat (Molteberg, Magnus, Bjorge, \& Nilsson, 1996; Cognat, Shepherd, Verrall, \& Stewart, 2012), and the amounts of hexanal and 2-pentylfuran increased only slightly during storage, reaching $8-18 \%$ of the values in the NHT oat samples after three months.

\section{Conclusions}

This study showed that accelerated solvent extraction at $40{ }^{\circ} \mathrm{C}$ was able to extract lipids, including epoxy and hydroxy fatty acids, from oat flours with good repeatability. The NVOFAs were separated from the neutral lipids using SPE and were further analysed using UHPLC-ELSD/MS. Epoxy and hydroxy fatty acids occurred in flours from NHT oat samples, and the quantities were higher in materials with unknown storage histories than in freshly milled ones. The quantities were insignificant in oat products that had been produced from HT grains, indicating the importance of POX activity to producing NVOFAs, which formed rapidly during storage in all NHT oat materials. Minor amounts of hydroxy and epoxy fatty acids could be detected soon after milling, and the amounts increased markedly during storage. In a controlled storage experiment, the highest total NVOFA amounts (1700-2000 $\mu \mathrm{g} / \mathrm{g})$ were reached in two months and then levelled off. In addition, there was a clear trend in the NVOFA profiles: at the beginning, the major products were epoxy fatty acids, after which the contents of hydroxy fatty acids began to increase. Epoxy fatty acids and 
493 hydroxy fatty acids were the primary products of POX formed by intermolecular oxygen transfer.

494 The quantities of both these products and of the hydrolysis products of epoxides, i.e. dihydroxy

495 fatty acids, began to increase only after extensive oxidation and remained at low levels. It could be

496 that off-flavours are related not only to the total amount of NVOFAs but also to the distribution of

497 the products. The importance of lipid-degrading enzymes was further supported by the fact that

498 formation of NVOFAs in line with FFAs, which are produced by lipase, and to that of

499 hydroperoxides, which are produced either by LOX or autoxidation. Finally, inactivating the

500 enzymes using HT significantly retarded all lipid degradation reactions.

501

502 Acknowledgements

503 The authors would like to thank Miikka Olin for his kindly technical assistances, Raisio Group and 504 Fazer for providing the oat samples. We would also thank CSC for funding the Ph.D studies of Z. 505 Yang. 


\section{References}

Baur, C., Grosch, W., Wieser, H., \& Jugel, H. (1977). Enzymatic Oxidation of Linoleic-Acid Formation of Bitter Tasting Fatty-Acids. Zeitschrift Fur Lebensmittel-Untersuchung UndForschung, 164(3), 171-176.

Benaragama, I., Meesapyodsuk, D., Beattie, A. D., \& Qiu, X. (2017). Identification and functional analysis of new peroxygenases in oat. Planta, 246(4), 711-719.

Biermann, U., Wittmann, A., \& Grosch, W. (1980). Occurrence of Bitter Hydroxy Fatty-Acids in Oat and Wheat. Fette Seifen Anstrichmittel, 82(6), 236-240.

Blée, E. (1998). Phytooxylipins and plant defense reactions. Progress in Lipid Research, 37(1), 33 72.

Cognat, C., Shepherd, T., Verrall, S. R., \& Stewart, D. (2012). Comparison of two headspace sampling techniques for the analysis of off-flavour volatiles from oat based products. Food Chemistry, 134(3), 1592-1600.

Damerau, A., Kamlang-ek, P., Moisio, T., Lampi, A. M., \& Piironen, V. (2014). Effect of SPME extraction conditions and humidity on the release of volatile lipid oxidation products from spray-dried emulsions. Food Chemistry, 157, 1-9.

Decker, E. A., Rose, D. J., \& Stewart, D. (2014). Processing of oats and the impact of processing operations on nutrition and health benefits. British Journal of Nutrition, 112, S58-S64.

Doehlert, D. C., Angelikousis, S., \& Vick, B. (2010). Accumulation of Oxygenated Fatty Acids in Oat Lipids During Storage. Cereal Chemistry, 87(6), 532-537.

Günther-Jordanland, K., Dawid, C., Dietz, M., \& Hofmann, T. (2016). Key phytochemicals contributing to the bitter off-taste of oat (Avena sativa L.). Journal of Agricultural and Food Chemistry, 64, 9639-9652. 
Hamberg, M., \& Hamberg, G. (1996). Peroxygenase-catalyzed fatty acid epoxidation in cereal seeds - Sequential oxidation of linoleic acid into 9(S),12(S),13(S)-trihydroxy-10(E)octadecenoic acid. Plant Physiology, 110(3), 807-815.

Hanano, A., Burcklen, M., Flenet, M., Ivancich, A., Louwagie, M., Garin, J., \& Blée, E. (2006). Plant seed peroxygenase is an original heme-oxygenase with an EF-hand calcium binding motif. Journal of Biological Chemistry, 281(44), 33140-33151.

Heimann, W., \& Klaiber, V. (1977). Hydroperoxide Degradation by Oat Isomerase - Investigation of Reaction-Mechanism. Zeitschrift Fur Lebensmittel-Untersuchung Und-Forschung, $165(3), 140-143$.

Ho, C. T., \& Chen, Q. Y. (1994). Lipids in Food Flavors - an Overview. Lipids in Food Flavors, 558, chapter 1 (pp. 2-14). Washington, DC: American Chemical Society.

Huang, F. C., \& Schwab, W. (2012). Overexpression of hydroperoxide lyase, peroxygenase and epoxide hydrolase in tobacco for the biotechnological production of flavours and polymer precursors. Plant Biotechnology Journal, 10(9), 1099-1109.

Jenske, R., \& Vetter, W. (2007). Highly selective and sensitive gas chromatography-electroncapture negative-ion mass spectrometry method for the indirect enantioselective identification of 2- and 3-hydroxy fatty acids in food and biological samples. Journal of Chromatography A, 1146(2), 225-231.

Lampi, A.-M., Damerau, A., Li, J., Moisio, T., Partanen, R., Forssell, P., \& Piironen, V. (2015). Changes in lipids and volatile compounds of oat flours and extrudates during processing and storage. Journal of Cereal Science, 62, 102-109.

Lehtinen, P., \& Kaukovirta-Norja, A. (2011). Oat lipids, enzymes, and quality. In F. H. Webster, \& P. J. Wood (Eds.), Oats: Chemistry and technology, second edition (pp. 143-156). St. Paul: AACC International, Int. 
Leonova, S., Shelenga, T., Hamberg, M., Konarev, A. V., Loskutov, I., \& Carlsson, A. S. (2008). Analysis of oil composition in cultivars and wild species of oat (Avena sp.). Journal of Agricultural and Food Chemistry, 56(17), 7983-7991.

Levandi, T., Püssa, T., Vaher, M., Toomik, P., \& Kaljurand, M. (2009). Oxidation products of free polyunsaturated fatty acids in wheat varieties. European Journal of Lipid Science and Technology, 111(7), 715-722.

Molteberg, E. L., Magnus, E. M., Bjorge, J. M., \& Nilsson, A. (1996). Sensory and chemical studies of lipid oxidation in raw and heat-treated oat flours. Cereal Chemistry, 73(5), 579-587.

Murphy, R. C. (2015). Tandem mass spectrometry of lipids: Molecular analysis of complex lipids. Cambridge: Royal Society of Chemistry.

Newman, J. W., Watanabe, T., \& Hammock, B. D. (2002). The simultaneous quantification of cytochrome P450 dependent linoleate and arachidonate metabolites in urine by HPLCMS/MS. Journal of Lipid Research, 43(9), 1563-1578.

Orellana-Coca, C., Adlercreutz, D., Andersson, M. M., Mattiasson, B., \& Hatti-Kaul, R. (2005). Analysis of fatty acid epoxidation by high performance liquid chromatography coupled with evaporative light scattering detection and mass spectrometry. Chemistry and Physics of Lipids, 135, 189-199.

Piazza, G. J., Foglia, T. A., \& Nuñez, A. (1999). Preparation of fatty epoxy alcohols using oat seed peroxygenase in nonaqueous media. Journal of the American Oil Chemists Society, 76(5), $551-555$.

Schneider, C., Schreier, P., \& Herderich, M. (1997). Analysis of lipoxygenase-derived fatty acid hydroperoxides by electrospray ionization tandem mass spectrometry. Lipids, 32(3), 331336.

Simsek, S., \& Doehlert, D. C. (2014). Oxygenated fatty acids isolated from wheat bran slurries. Int Journal of Food Science \& Nutrition, 65(7), 803-808. 
578 Yao, L., \& Schaich, K. M. (2014). Accelerated Solvent Extraction Improves Efficiency of Lipid Removal from Dry Pet Food While Limiting Lipid Oxidation. Journal of the American Oil Chemists' Society, 92(1), 141-151.

581 Yang, Z., Piironen, V., \& Lampi, A. M. (2017). Lipid-modifying enzymes in oat and faba bean. Food Research International, 100(1), 335-343.

583 Yang, N. Y., Yang, Y. F., \& Li, K. (2013). Analysis of Hydroxy Fatty Acids from the Pollen of 584 Brassica campestris L. var. oleifera DC. by UPLC-MS/MS. Journal of Pharmaceutics, 2013,874875 .

586 Yuan, Z. X., Majchrzak-Hong, S., Keyes, G. S., Iadarola, M. J., Mannes, A. J., \& Ramsden, C. E. (2018). Lipidomic profiling of targeted oxylipins with ultra-performance liquid chromatography-tandem mass spectrometry. Analytical and Bioanalytical Chemistry, 410(23), 6009-6029. 
591 Fig. 1. UHPLC-ELSD chromatogram of oxidised fatty acids from oat lipid extracts after SPE 592 fractionation. $\mathrm{C} 1$ = 12,13-dihydroxy-octadec-9-enoic acid; C2 = a mixture of 13-hydroxy-9,10593 epoxy-octadec-11-enoic acid and 9-hydroxy-12,13-epoxy-octadec-10-enoic acid; C3 = 13-hydroxy594 octadeca-9,11-dienoic acid; C4 = 9-hydroxy-octadeca-10,12-dienoic acid; C5 = 12,13-epoxy595 octadec-9-enoic acid; C6 = 9,10-epoxy-octadec-12-enoic acid; and C7 = 9,10-epoxyoctadecanoic 596 acid.

597

598 Fig. 2. Mass spectra (a-g) of the compounds C1-C7 as presented in Figure 1, produced using 599 UHPLC-Q-TOF with ESI ionisation in the negative mode.

600

601 Fig. 3. Contents of a) free fatty acids (mg/g flour) and b) TAGs (mg/g flour) in the flours made 602 from NHT and HT oat grains during storage at $40{ }^{\circ} \mathrm{C}$.

603

604 Fig. 4. Peak areas of a) hexanal and b) 2-pentylfuran in counts per second of the flours made from 605 NHT and HT oat grains during storage at $40{ }^{\circ} \mathrm{C}$. 
607 Table 1. Contents ( $\mu \mathrm{g} / \mathrm{g}$ flour) of non-volatile lipid oxidation products*

$608 *$ a) products from NHT oat grains stored at $35^{\circ} \mathrm{C}, \mathrm{b}$ ) flours from NHT oat grains stored at $40{ }^{\circ} \mathrm{C}$ 609 and c) fours from HT oat grains stored at $40{ }^{\circ} \mathrm{C}(\mathrm{n}=3$, mean \pm standard deviation). Samples in a) 610 were O-HT-F1, O-HT-F2, O-NHT-P, F-NHT-F1 and F-NHT-F2. Samples in b) were NHT-IS, 611 NHT-CV1 and NHT-CV2. Samples in c) were HT-IS, HT-CV1 and HT-CV2. Compounds analysed 612 were C1: 12,13-dihydroxy-octadec-9-enoic acid; C2: a mixture of 13-hydroxy-9,10-epoxy-octadec613 11-enoic acid and 9-hydroxy-12,13-epoxy-octadec-10-enoic acid; C3: 13-hydroxy-octadeca-9,11614 dienoic acid; C4: 9-hydroxy-octadeca-10,12-dienoic acid; C5: 12,13-epoxy-octadec-9-enoic acid; 615 C6: 9,10-epoxy-octadec-12-enoic acid; and C7: 9,10-epoxyoctadecanoic acid. 


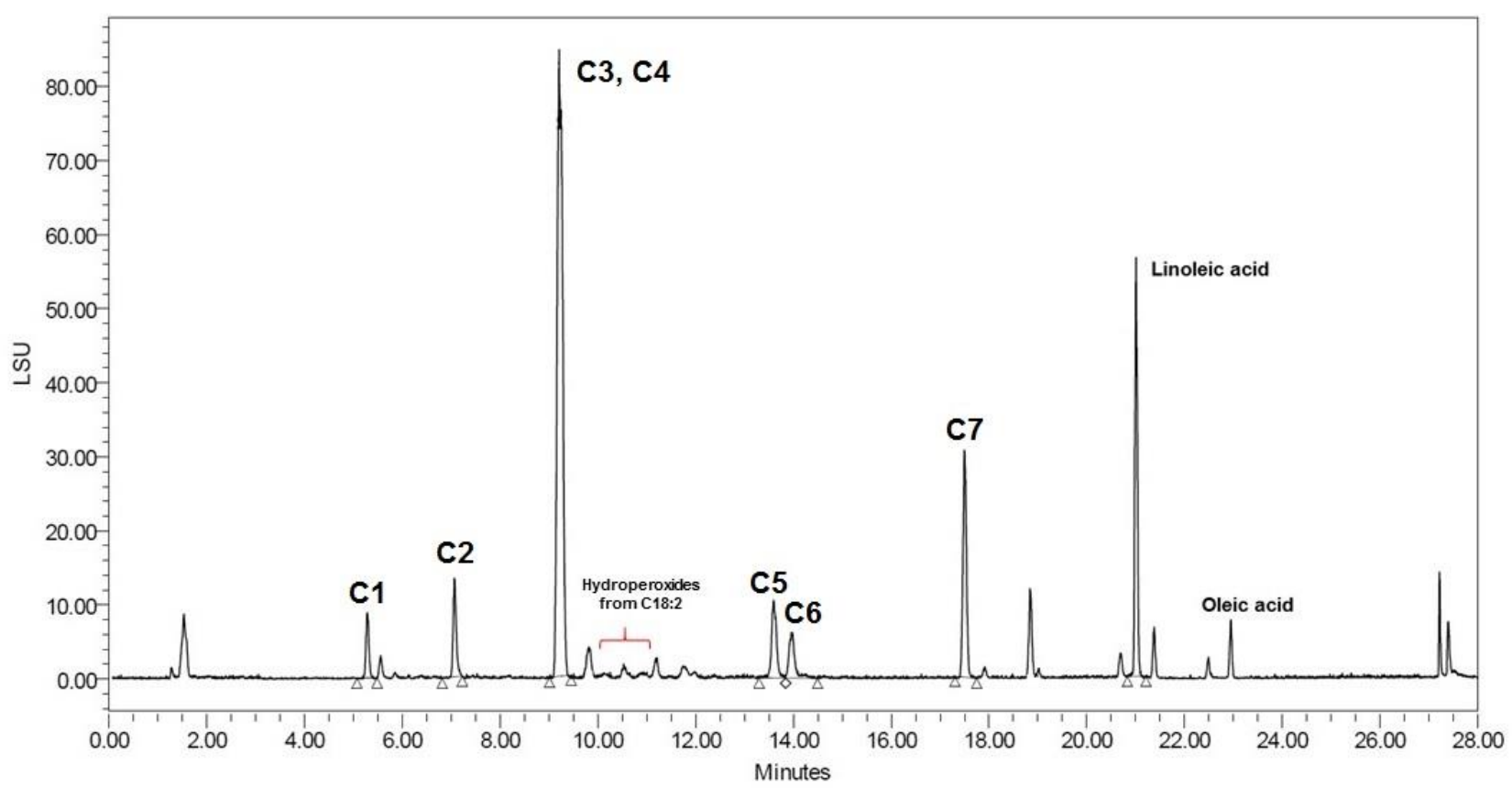

Fig. 1. 

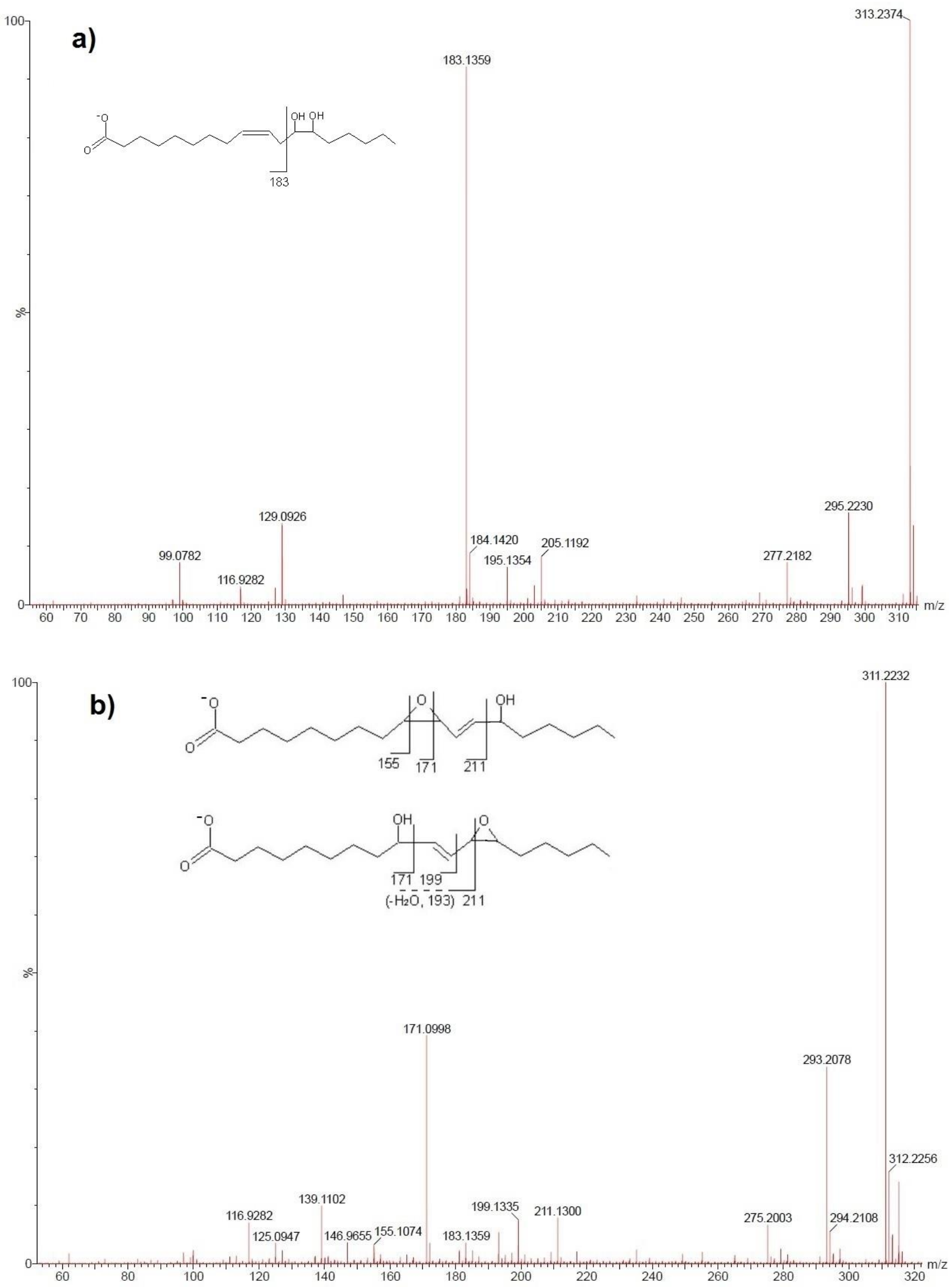

Fig. 2. 

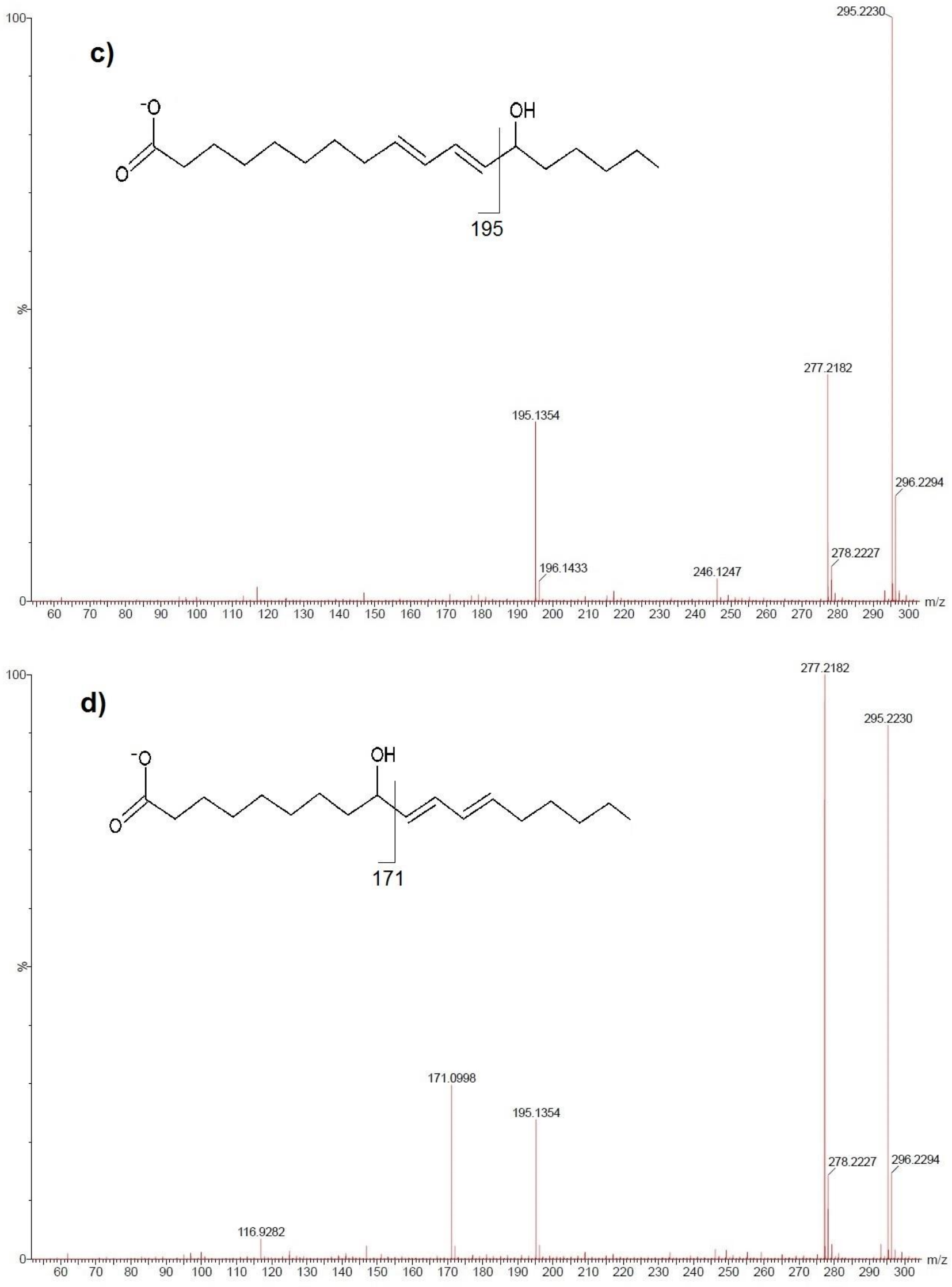

Fig. 2. (Continued). 

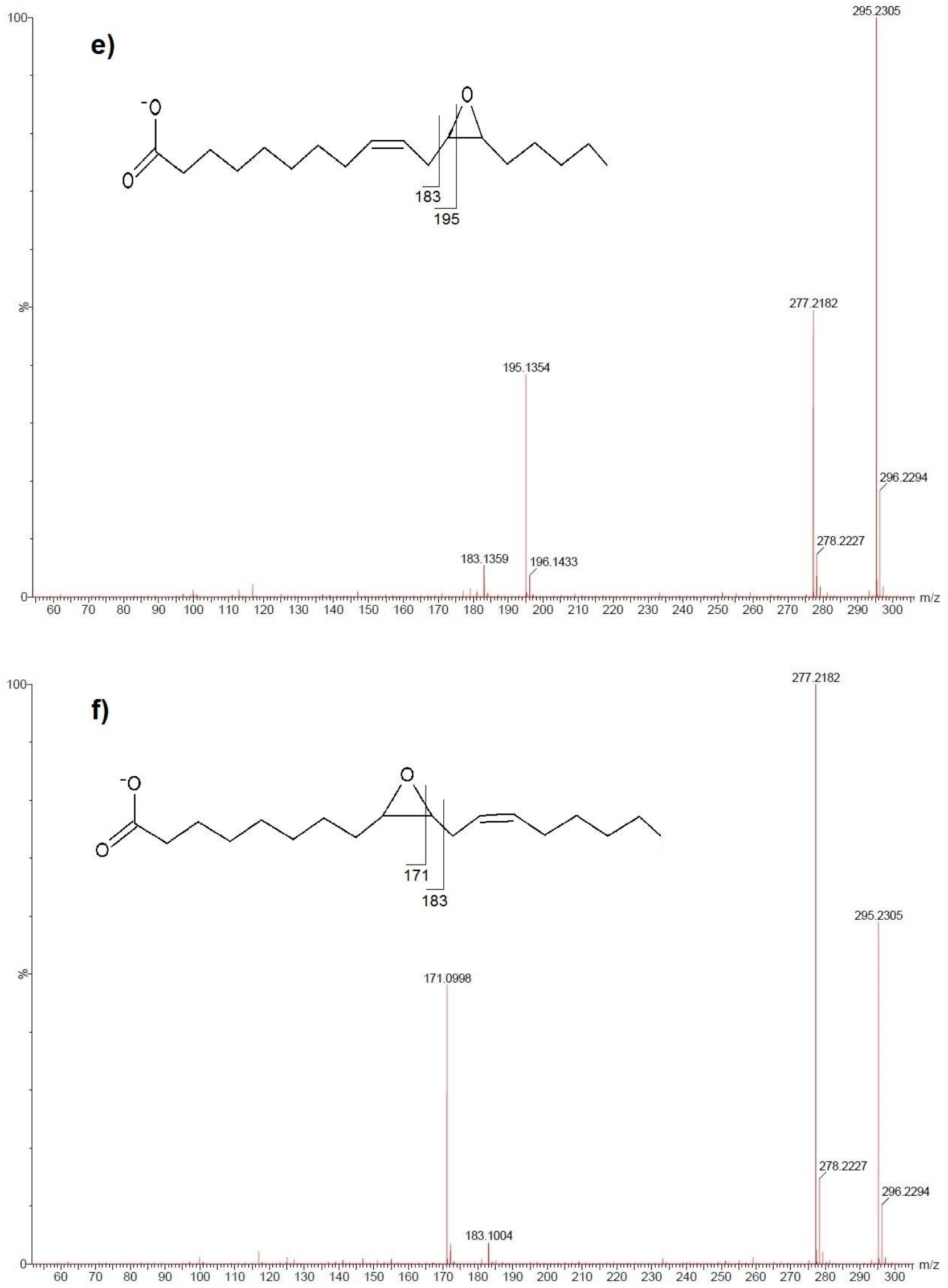

Fig. 2. (Continued). 


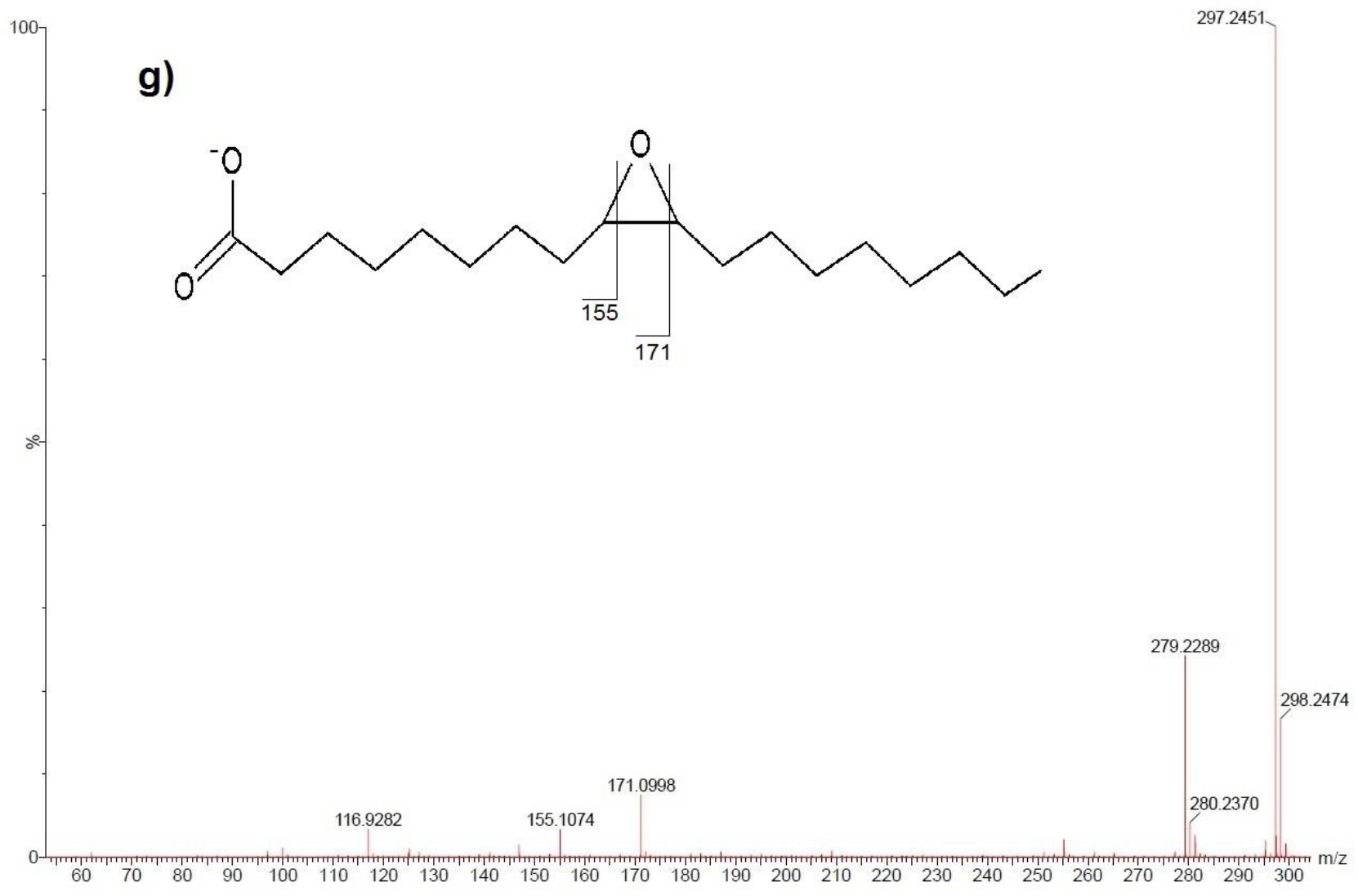

Fig. 2. (Continued). 

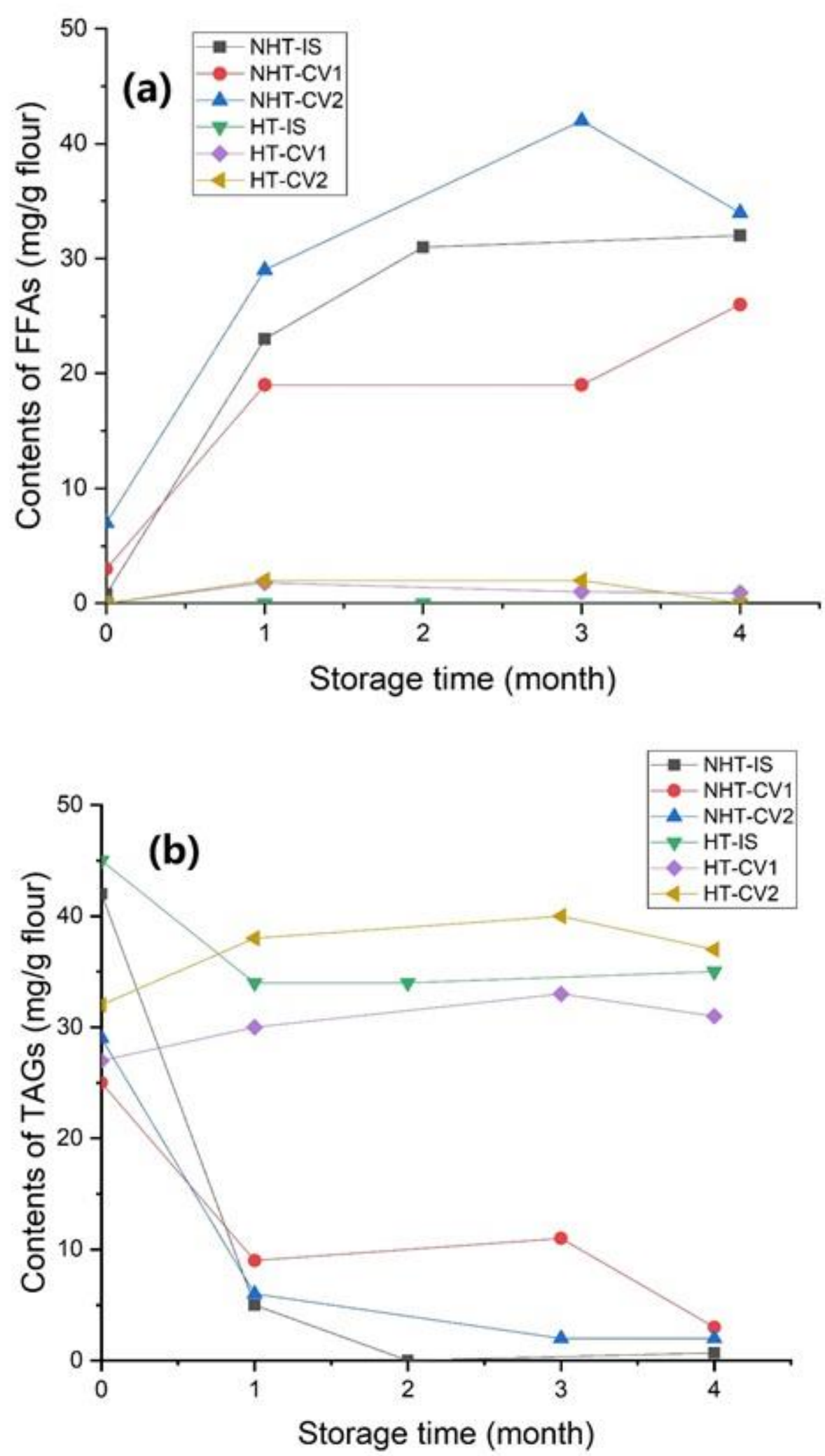

Fig. 3. 

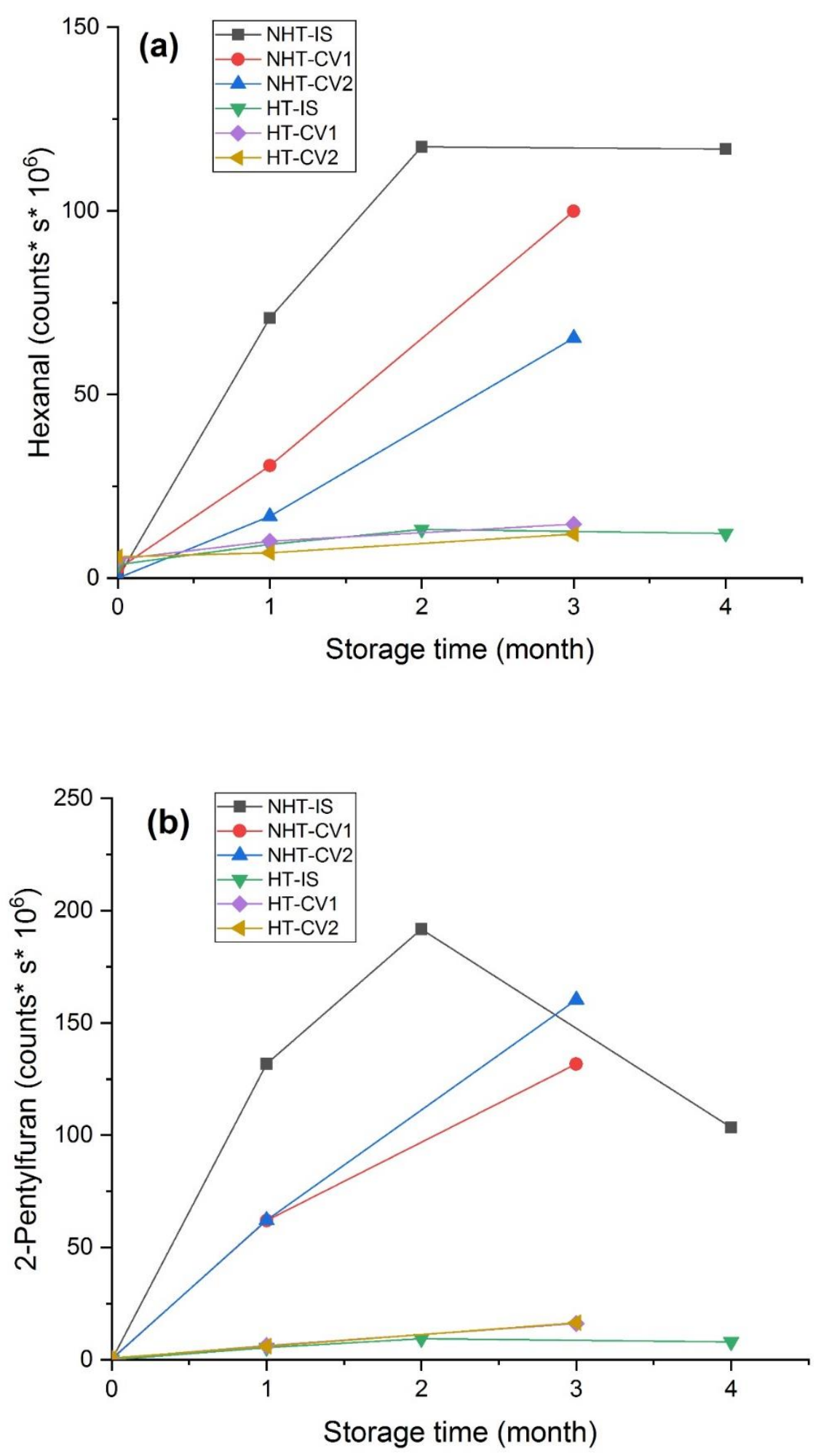

Fig. 4. 
Table 1.

a)

\begin{tabular}{|c|c|c|c|c|c|c|c|c|}
\hline & \multirow{2}{*}{ Oat samples } & \multicolumn{7}{|c|}{ Non-volatile lipid oxidation products in oat ( $\mu \mathrm{g} / \mathrm{g}$ flour) } \\
\hline & & $\mathrm{C} 1$ & $\mathrm{C} 2$ & $\mathrm{C} 3+\mathrm{C} 4$ & $\mathrm{C} 5$ & C6 & $\mathrm{C7}$ & Total \\
\hline & O-HT-F1 & nd & nd & nd & nd & nd & nd & nd \\
\hline & O-HT-F2 & nd & nd & nd & nd & nd & nd & nd \\
\hline & O-NHT-P & nd & $54 \pm 4$ & $565 \pm 23$ & $58 \pm 2$ & $54 \pm 1$ & $69 \pm 2$ & $800 \pm 32$ \\
\hline Oat samples & $\begin{array}{l}\text { Sampling time } \\
\left(35^{\circ} \mathrm{C} \text { storage }\right)\end{array}$ & & & & & & & \\
\hline \multirow{5}{*}{ F-NHT-F1 } & 0 weeks & nd & nd & $119 \pm 3$ & $66 \pm 2$ & $61 \pm 2$ & $104 \pm 4$ & $350 \pm 11$ \\
\hline & 6 weeks & $58 \pm 0.4$ & $57 \pm 0.3$ & $667 \pm 8$ & $134 \pm 5$ & $116 \pm 5$ & $298 \pm 10$ & $1330 \pm 29$ \\
\hline & 12 weeks & $53 \pm 2$ & $64 \pm 1$ & $665 \pm 5$ & $139 \pm 4$ & $115 \pm 4$ & $331 \pm 11$ & $1367 \pm 27$ \\
\hline & 18 weeks & $51 \pm 1$ & $73 \pm 2$ & $732 \pm 23$ & $347 \pm 3$ & $349 \pm 2$ & $881 \pm 37$ & $2433 \pm 68$ \\
\hline & 24 weeks & $55 \pm 0.2$ & $81 \pm 4$ & $756 \pm 7$ & $61 \pm 1$ & $53 \pm 1$ & $64 \pm 1$ & $1070 \pm 14$ \\
\hline \multirow{5}{*}{ F-NHT-F2 } & 0 weeks & nd & nd & $90 \pm 2$ & $55 \pm 1$ & $55 \pm 3$ & $84 \pm 1$ & $284 \pm 7$ \\
\hline & 6 weeks & $73 \pm 1$ & $97 \pm 5$ & $540 \pm 8$ & $92 \pm 1$ & $79 \pm 1$ & $142 \pm 3$ & $1023 \pm 19$ \\
\hline & 12 weeks & $70 \pm 0.4$ & $152 \pm 9$ & $475 \pm 23$ & $183 \pm 3$ & $160 \pm 6$ & $404 \pm 7$ & $1444 \pm 48$ \\
\hline & 18 weeks & $95 \pm 5$ & $523 \pm 16$ & $257 \pm 9$ & $169 \pm 3$ & $177 \pm 2$ & $505 \pm 8$ & $1726 \pm 43$ \\
\hline & 24 weeks & $95 \pm 7$ & $495 \pm 4$ & $223 \pm 4$ & $114 \pm 2$ & $105 \pm 2$ & $196 \pm 1$ & $1228 \pm 20$ \\
\hline
\end{tabular}




\begin{tabular}{|c|c|c|c|c|c|c|c|c|}
\hline \multirow[t]{2}{*}{ Oat samples } & \multirow{2}{*}{$\begin{array}{l}\text { Sampling time } \\
\left(40^{\circ} \mathrm{C} \text { storage }\right)\end{array}$} & \multicolumn{7}{|c|}{ Non-volatile lipid oxidation products in NHT oat ( $\mu \mathrm{g} / \mathrm{g}$ flour) } \\
\hline & & $\mathrm{C} 1$ & $\mathrm{C} 2$ & $\mathrm{C} 3+\mathrm{C} 4$ & $\mathrm{C} 5$ & C6 & $\mathrm{C} 7$ & Total \\
\hline \multirow{5}{*}{ NHT-IS } & Freshly milled & nd & nd & nd & bdl & bdl & $57 \pm 2$ & $57 \pm 2$ \\
\hline & 1 month & nd & nd & $328 \pm 8$ & $311 \pm 5$ & $303 \pm 5$ & $699 \pm 7$ & $1641 \pm 25$ \\
\hline & 2 months & $60 \pm 2$ & $69 \pm 1$ & $466 \pm 3$ & $243 \pm 17$ & $219 \pm 13$ & $631 \pm 19$ & $1688 \pm 55$ \\
\hline & 3 months & $76 \pm 1$ & $97 \pm 3$ & $564 \pm 7$ & $132 \pm 17$ & $108 \pm 12$ & $426 \pm 58$ & $1403 \pm 98$ \\
\hline & 4 months & $46 \pm 2$ & $67 \pm 4$ & $409 \pm 27$ & $50 \pm 1$ & $49 \pm 1$ & $71 \pm 1$ & $692 \pm 36$ \\
\hline \multirow{5}{*}{ NHT-CV1 } & Freshly milled & bdl & bdl & bdl & bdl & bdl & bdl & bdl \\
\hline & 1 month & bdl & bdl & $424 \pm 3$ & $167 \pm 12$ & $141 \pm 11$ & $382 \pm 0.07$ & $1114 \pm 26$ \\
\hline & 2 months & bdl & bdl & $348 \pm 10$ & $352 \pm 25$ & $387 \pm 14$ & $833 \pm 59$ & $1920 \pm 108$ \\
\hline & 3 months & bdl & bdl & $601 \pm 14$ & $252 \pm 16$ & $187 \pm 13$ & $471 \pm 16$ & $1511 \pm 59$ \\
\hline & 4 months & $65 \pm 1$ & $121 \pm 5$ & $687 \pm 14$ & $68 \pm 1$ & $61 \pm 0.3$ & $107 \pm 6$ & $1109 \pm 27$ \\
\hline \multirow{5}{*}{ NHT-CV2 } & Freshly milled & nd & nd & $49 \pm 0.2$ & nd & nd & $63 \pm 0.4$ & $112 \pm 1$ \\
\hline & 1 month & $47 \pm 1$ & $52 \pm 0.4$ & $441 \pm 3$ & $106 \pm 3$ & $97 \pm 2$ & $285 \pm 11$ & $1028 \pm 20$ \\
\hline & 2 months & $51 \pm 6$ & $63 \pm 9$ & $491 \pm 17$ & $297 \pm 8$ & $295 \pm 26$ & $789 \pm 34$ & $1986 \pm 100$ \\
\hline & 3 months & $80 \pm 5$ & $113 \pm 8$ & $716 \pm 14$ & $200 \pm 7$ & $159 \pm 9$ & $533 \pm 29$ & $1801 \pm 72$ \\
\hline & 4 months & $66 \pm 2$ & $99 \pm 1$ & $637 \pm 7$ & $55 \pm 1$ & $53 \pm 0.2$ & $76 \pm 0.4$ & $986 \pm 12$ \\
\hline
\end{tabular}


c)

\begin{tabular}{|c|c|c|c|c|c|c|c|c|}
\hline \multirow[t]{2}{*}{ Oat samples } & \multirow{2}{*}{$\begin{array}{l}\text { Sampling time } \\
\left(40^{\circ} \mathrm{C} \text { storage }\right)\end{array}$} & \multicolumn{7}{|c|}{ Non-volatile lipid oxidation products in HT oat ( $\mu \mathrm{g} / \mathrm{g}$ flour) } \\
\hline & & $\mathrm{C} 1$ & $\mathrm{C} 2$ & $\mathrm{C} 3+\mathrm{C} 4$ & $\mathrm{C} 5$ & C6 & $\mathrm{C} 7$ & Total \\
\hline \multirow{5}{*}{ HT-IS } & Freshly milled & nd & nd & nd & nd & nd & nd & nd \\
\hline & 1 month & nd & nd & nd & $61 \pm 2$ & $55 \pm 1$ & $62 \pm 3$ & $178 \pm 6$ \\
\hline & 2 months & nd & nd & nd & $53 \pm 1$ & $51 \pm 1$ & $61 \pm 2$ & $165 \pm 4$ \\
\hline & 3 months & nd & nd & nd & $58 \pm 1$ & $52 \pm 1$ & $61 \pm 3$ & $171 \pm 5$ \\
\hline & 4 months & nd & nd & bdl & $54 \pm 0.3$ & $52 \pm 0.2$ & $59 \pm 2$ & $165 \pm 3$ \\
\hline \multirow{5}{*}{ HT-CV1 } & Freshly milled & nd & nd & nd & nd & nd & nd & nd \\
\hline & 1 month & nd & nd & bdl & bdl & bdl & bdl & bdl \\
\hline & 2 months & nd & nd & bdl & $56 \pm 1$ & $53 \pm 1$ & $70 \pm 3$ & $179 \pm 5$ \\
\hline & 3 months & nd & nd & $48 \pm 2$ & $67 \pm 2$ & $60 \pm 1$ & $78 \pm 5$ & $253 \pm 10$ \\
\hline & 4 months & nd & nd & $44 \pm 1$ & bdl & bdl & $54 \pm 1$ & $98 \pm 2$ \\
\hline \multirow{5}{*}{ HT-CV2 } & Freshly milled & nd & nd & nd & nd & nd & nd & nd \\
\hline & 1 month & nd & nd & bdl & bdl & bdl & $60 \pm 2$ & $60 \pm 2$ \\
\hline & 2 months & nd & nd & bdl & $60 \pm 1$ & $57.6 \pm 0.1$ & $93.4 \pm 0.2$ & $210 \pm 1$ \\
\hline & 3 months & nd & nd & $65 \pm 6$ & $93 \pm 9$ & $76 \pm 4$ & $164 \pm 18$ & $398 \pm 37$ \\
\hline & 4 months & nd & nd & $44 \pm 1$ & bdl & bdl & $54 \pm 0.4$ & $98 \pm 1$ \\
\hline
\end{tabular}

Where, nd: not detected; bdl: below detection limit. 